\title{
Small Intestinal Bacterial Overgrowth Diagnosed by Glucose Hydrogen Breath Test in Post-cholecystectomy Patients
}

\author{
Hea Jung Sung, ${ }^{1}$ Chang-Nyol Paik, ${ }^{1 *}$ Woo Chul Chung, ${ }^{1}$ Kang-Moon Lee, ${ }^{1}$ Jin-Mo Yang, ${ }^{1}$ and Myung-Gyu Choi ${ }^{2}$ \\ ${ }^{1}$ Division of Gastroenterology, Department of Internal Medicine, St. Vincent's Hospital, College of Medicine, The Catholic University of Korea, \\ Seoul, Korea; and ${ }^{2}$ Division of Gastroenterology, Department of Internal Medicine, Seoul St. Mary's Hospital, College of Medicine, The Catholic \\ University of Korea, Seoul, Korea
}

\section{Background/Aims}

Patients undergoing cholecystectomy may have small intestinal bacterial overgrowth (SIBO). We investigated the prevalence and characteristics of SIBO in patients with intestinal symptoms following cholecystectomy.

\section{Methods}

Sixty-two patients following cholecystectomy, 145 with functional gastrointestinal diseases (FGIDs), and 30 healthy controls undergoing hydrogen $\left(\mathrm{H}_{2}\right)$-methane $\left(\mathrm{CH}_{4}\right)$ glucose breath test $(\mathrm{GBT})$ were included in the study. Before performing $\mathrm{GBT}$, all patients were interrogated using bowel symptom questionnaire. The positivity to GBT indicating the presence of SIBO, gas types and bowel symptoms were surveyed.

\section{Results}

Post-cholecystectomy patients more often had SIBO as evidenced by a positive $(+)$ GBT than those with FGID and controls $(29 / 62,46.8 \%$ vs $38 / 145,26.2 \%$ vs $4 / 30,13.3 \%$, respectively; $P=0.010)$. In the gas types, the GBT $\left(\mathrm{H}_{2}\right)+$ post-cholecystectomy patients was significantly higher than those in FGIDs patients $(P=0.017)$. Especially, positivity to fasting $G B T\left(H_{2}\right)$ among the GBT $\left(\mathrm{H}_{2}\right)+$ post-cholecystectomy patients was high, as diagnosed by elevated fasting $\mathrm{H}_{2}$ level. The GBT+ group had higher symptom scores of significance or tendency in abdominal discomfort, bloating, chest discomfort, early satiety, nausea, and tenesmus than those of the GBT negative group. The status of cholecystectomy was the only significant independent factor for predicting SIBO.

\section{Conclusions}

The SIBO with high levels of baseline $\mathrm{H}_{2}$ might be the important etiologic factor of upper $\mathrm{Gl}$ symptoms for post-cholecystectomy patients.

(J Neurogastroenterol Motil 2015;21:545-551)

Key Words

Breath test; Cholecystectomy; Glucose

Received: February 4, 2015 Revised: May 12, 2015 Accepted: May 15, 2015

(c) This is an Open Access article distributed under the terms of the Creative Commons Attribution Non-Commercial License (http://creativecommons. org/licenses/by-nc/4.0) which permits unrestricted non-commercial use, distribution, and reproduction in any medium, provided the original work is properly cited.

${ }^{*}$ Correspondence: Chang-Nyol Paik, MD, PhD

Division of Gastroenterology, Department of Internal Medicine, St. Vincent's Hospital, College of Medicine, The Catholic University of Korea, 93, Jungbu-Daero, Paldal-Gu, Suwon, Gyeonggi-Do 16247, Korea

Financial support: None. Tel: +82-31-249-8374, Fax: +82-31-253-8898, E-mail: cmcu@catholic.ac.kr

Conflicts of interest: None.

Author contributions: Hea Jung Sung and Chang-Nyol Paik collected the data, designed the study, drafted and revised the manuscript, and approved the final version of the manuscript; Woo Chul Chung, Kang-Moon Lee, and Jin-Mo Yang collected and analyzed data; and Myung-Gyu Choi supervised the study and extensively revised the manuscript. 


\section{Introduction}

Significant number of patients experience post-cholecystectomy syndrome, as laparoscopic cholecystectomy has been increased. Postcholecystectomy syndrome is defined as the presence of abdominal symptoms after surgical removal of the gallbladder. This usually takes the form of upper abdominal pain and dyspepsia with or without jaundice. Most common causes of postcholecystectomy syndrome has been known as extrabiliary disorders such as reflux esophagitis, peptic ulceration, irritable bowel syndrome (IBS) or chronic pancreatitis. ${ }^{1}$ Recently, a role for enteric bacteria was suggested to be one of the etiologic factors for the development of symptoms in patients with cholecystectomy. ${ }^{2}$

The concept of a possible association between small intestinal bacterial overgrowth (SIBO) and the postcholecystectomy status has some logic as several intestinal symptoms of these 2 conditions are similar. Cholecystectomy may induce the several physiological changes in the upper gastrointestinal (GI) tract which may account for the persistence of symptoms or the development of new symptoms after gallbladder removal. Bile acids have antimicrobial effects in the intestine. Therefore, cholecystectomy may also modify the metabolic activity of the intestinal bacteria. ${ }^{3}$

SIBO is caused by an abnormally increased number of bacteria in the small bowel and associated with various intestinal symptoms or functional gastrointestinal disorders (FGIDs). Some believe jejunal aspirate and culture as the gold standard to establish SIBO. To detect the bacteria, the specificity of glucose breath test (GBT) was $100 \%$ but its sensitivity was low as $27 \%$ to $56 \%$ on the basis of upper gut aspirate culture in the literature. However, there are still limitations of jejunal aspiration method which include lack of accessibility to the distal small intestine, potential for contamination during sampling, and possibility of false negative results, especially where obligate anaerobes are involved. Therefore, GBT became a simple alternative and acceptable method for diagnosis of SIBO. ${ }^{6,7}$ To our knowledge, there is limited data available for the evaluation on the relationship between SIBO and the intestinal symptoms in postcholecystectomy patients. The aim of this study is to evaluate the prevalence and characteristics of SIBO in patients with intestinal symptoms using GBT according to the history of cholecystectomy.

\section{Materials and Methods}

This study was approved by the Institutional Research Ethics Board of the Catholic University of Korea (no.VC14RISI0083) and adhered to the declaration of Helsinki.

\section{Study Populations}

We retrospectively reviewed a prospectively collected database for the consecutive outpatients who had undergone GBT for GI symptoms at a referral center, St. Vincent's Hospital Gastroenterology clinic, the Catholic University of Medicine. All patients that attended the clinic between December 2009 and March 2012 were included in this study. The patients over 18 years of age, who had a FGIDs without history of cholecystectomy, or who underwent laparoscopic cholecystectomy with gastrointestinal symptoms irrespective of FGIDs were enrolled. The patients with cholecystectomy underwent laparoscopic surgery due to acute cholecystitis with gallbladder stone at least 6 months before, and had no evidence of remnant stone at cystic duct or common bile duct by the blood chemistry or abdominal computed tomography scan. The patients with FGIDs were defined, according to the Rome III for the previous 3 months, with symptom onset at least 6 months prior to diagnosis. ${ }^{8,9}$ Patients were excluded if they had a history of diabetes mellitus, connective tissue disease, thyroid disease or gastrointestinal surgery except laparoscopic cholecystectomy. Other exclusion criteria included the following: taking antisecretory agent such as proton pump inhibitors or histamine $(\mathrm{H})_{2}$ receptor antagonists, antibiotics, probiotics, prokinetics, or narcotic use, probiotics, laxatives, bulking agent, or antidiarrheal drugs; having a gastrointestinal disease, renal insufficiency, liver disease, a major psychiatric disease, hearing impairment, masticatory dysfunction; having undergone colonoscopy within the previous 3 months; or having incomplete data. The GBTs in patients with cholecystectomy and FGIDs were compared with 30 historical healthy controls who were enrolled for determination of the normal values for GBT at the catholic university of Medicine in $2007 .^{10}$

\section{Assessment of Abdominal Symptoms}

The demographic data, the comorbidities, the concurrent use of drugs, the predominant bowel symptoms, and a bowel symptom questionnaire were routinely surveyed during the GBT for evaluating and making the diagnosis of patients with clinically 
suspected gastrointestinal disorders. Before performing GBT, all patients were asked using careful history taking and bowel symptom questionnaire. The questionnaire form included the Rome III criteria and additional questions with regard to severity of bowel symptoms, which has been validated in other studies. ${ }^{11,12} \mathrm{~A}$ total of 13 bowel symptoms were asked; abdominal discomfort/pain or cramps, hard or lumpy stools, loose or watery stools, straining during a bowel movement, having to rush to the toilet for a bowel movement (urgency), tenesmus, mucus in stool, passing mucus during a bowel movement, abdominal fullness/bloating or swelling, passing gas (flatus), heartburn or chest pain, feeling full soon after starting a meal, passing urine frequently, and nausea. The severity of symptoms was evaluated by the total symptom score, which was defined as the sum of the symptom frequency and bothersomeness scores. The frequency and bothersomeness of each symptom were assessed using a seven point scale from 0 (never) to 6 (always or extremely). The range of total symptom scores in each individual symptom was 0-12.

\section{Glucose Breath Test}

The GBT test was performed to diagnose SIBO after an overnight fast of at least 12 hours. Breath test started 30 minutes after mouth washing with $20 \mathrm{~mL}$ of $0.05 \%$ chlorhexidine mouth and water. Smoking and physical exercise were not allowed for 30 minutes before and during the test. Patients were instructed to take $50 \mathrm{~g}$ of glucose (DIASOL-S SOLN, Tae Joon Pharma, Seoul, Korea). The end expiratory breath samples were collected and breath hydrogen $\left(\mathrm{H}_{2}\right)$ and methane $\left(\mathrm{CH}_{4}\right)$ values were estimated at baseline, and then at every 10-minute intervals for 120 minutes. Duplicate samples were taken at each time with the equipment of the breath test (the Quintron SC breathtracker; Quintron Instrument Company, Milwaukee, WI, USA). The positivities to GBT for $\mathrm{H}_{2}\left(\mathrm{GBT}\left[\mathrm{H}_{2}\right]+\right)$ or $\mathrm{CH}_{4}(\mathrm{GBT}$ $\left.\left[\mathrm{CH}_{4}\right]+\right)$ indicating the diagnosis of SIBO were defined as (1) a baseline $\mathrm{H}_{2}$ or $\mathrm{CH}_{4}$ concentration of more than $15 \mathrm{ppm}^{13}$ or (2) increase in the breath $\mathrm{H}_{2}$ or $\mathrm{CH}_{4}$ concentration of more than 12 ppm above the baseline value within 60 minutes after ingestion of the glucose solution. ${ }^{11,14}$ The positivity including both GBT $\left(\mathrm{H}_{2}\right)+$ and GBT $\left(\mathrm{CH}_{4}\right)+$ status was classified as GBT (both) positive status.

\section{Statistical Methods}

The continuous data were expressed as mean \pm standard deviation and analyzed using independent samples $t$ tests with Levene's test for equality of variances, whereas the categorical variables were expressed as quantities and they were analyzed using $\chi 2$ tests or Fisher's exact test. Multiple stepwise logistic regression analysis was used to identify the independent factors associated with positivity to GBT. The data were performed by SPSS 21.0 software version. A $P$-value $<0.05$ was considered statistically significant.

\section{Results}

\section{Study Populations}

During study period, 314 consecutive patients underwent GBT due to intestinal symptom. Among them, 107 patients were

Table 1. Demographic Clinical Data of Patients With and Without Cholecystectomy

\begin{tabular}{|c|c|c|c|c|}
\hline & $\begin{array}{l}\text { Patients with cholecystectomy } \\
\qquad(\mathrm{n}=62)\end{array}$ & $\begin{array}{l}\text { Patients with FGIDs } \\
\qquad(\mathrm{n}=145)\end{array}$ & $\begin{array}{l}L \text {-test }{ }^{\mathrm{a}} \\
P \text {-value }\end{array}$ & $P$-value \\
\hline Age (year) & $53.27 \pm 15.27$ & $49.01 \pm 15.98$ & 0.65 & 0.08 \\
\hline Gender (male) & $37(60 \%)$ & $96(66 \%)$ & & 0.37 \\
\hline $\mathrm{BMI}\left(\mathrm{Kg} / \mathrm{m}^{2}\right)$ & $24.35 \pm 3.70$ & $23.42 \pm 13.61$ & 0.61 & 0.60 \\
\hline Positive breath test & $29(47 \%)$ & $38(26 \%)$ & & $<0.01$ \\
\hline $\mathrm{H}_{2}$ & $27(43.5 \%)$ & $29(20 \%)$ & & 0.02 \\
\hline $\mathrm{CH}_{4}$ & $2(3.2 \%)$ & $9(6 \%)$ & & 0.37 \\
\hline Both & 0 & 0 & & \\
\hline Diagnosis of IBS & $16(26 \%)$ & $41(28 \%)$ & & 0.82 \\
\hline Total symptom score & $46.69 \pm 27.12$ & $50.45 \pm 29.25$ & 0.29 & 0.39 \\
\hline Frequency score & $25.53 \pm 13.30$ & $27.16 \pm 14.23$ & 0.51 & 0.45 \\
\hline Bothersome score & $21.16 \pm 14.37$ & $23.29 \pm 15.64$ & 0.28 & 0.36 \\
\hline
\end{tabular}

${ }^{a}$ Levene's test for Equality of Variances for continuous variables.

BMI, body mass index; FGIDs, functional gastrointestinal disorders; BMI, body mass index; IBS, irritable bowel syndrome. 
excluded because of history of diabetes $(n=34)$, abdominal surgery $(n=29)$, peptic ulcer $(n=17)$, history of drugs $(n=8)$, recurrent common bile duct stone $(\mathrm{n}=5)$, thyroid disease $(\mathrm{n}=$ $4)$, pancreatitis $(n=2)$, pregnancy $(n=1)$, renal insufficiency ( $n$ $=1)$, hepatitis $(\mathrm{n}=1)$, liver cirrhosis $(\mathrm{n}=1)$ gastric cancer $(\mathrm{n}$ $=1)$, and incompletely filled questionnaire $(\mathrm{n}=3)$. Sixty-two postcholecystectomy patients with intestinal symptoms and 145 FGIDs patients were finally enrolled. No significant differences in age, gender, body mass index (BMI), and the prevalence of IBS were found between post cholecystectomy patients and FGIDs patients (Table 1).

\section{Comparison of the Glucose Breath Test in Patients With and Without Cholecystectomy}

The positivity to GBT was significantly frequent in patients

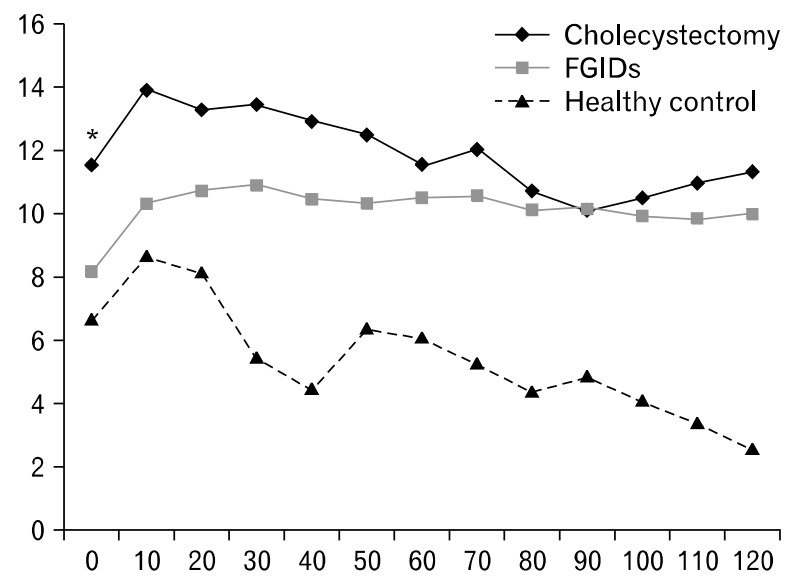

Figure 1. The profiles of glucose hydrogen breath test in patients with postcholecystectomy, functional gastrointestinal disorders (FGIDs), and healthy control. The mean level of basal breath $\mathrm{H}_{2}$ in postcholecystectomy patients was higher than that in FGIDs patients $\left({ }^{*} P<\right.$ $0.05)$. with cholecystectomy $(29 / 62,46.8 \%)$ than those with FGIDs $(38 / 145,26.2 \% ; P<0.01)$ (Table 1$)$, or those in healthy controls $(4 / 30,13.3 \% ; P<0.01)$. At all time point, breath $\mathrm{H}_{2}$ values were lower among patients with FGIDs and controls compared to those following cholecystectomy. However, no differences were shown in single time points except 0 minute between the patients following cholecystectomy and those with FGIDs. The mean level of basal breath $\mathrm{H}_{2}$ in postcholecystectomy patients was higher than that in FGIDs patients $(11.60 \pm 11.26$ vs $8.14 \pm 10.60 ; P=0.036$ ) (Fig. 1). In the gas types, the GBT $\left(\mathrm{H}_{2}\right)+$ status in postcholecystectomy patients was significantly higher than that in FGID patients ( $43.5 \%$ vs $20.0 \%, P=0.017$ ). No significant differences were observed in frequency score, bothersome score, total symptom score (Table 1), and individual symptoms in both groups.

\section{Characteristics of the Patients With Cholecystectomy According to the Positivity to Glucose Breath Test}

There were no difference between GBT + group (29/62) and GBT - group (33/62) of post cholecystectomy patients regarding age, gender, BMI, and the presence of IBS. Among postcholecystectomy patients, 27 (43.5\%), 2 (3.2\%), and 0 (0.0\%) were in the GBT $\left(\mathrm{H}_{2}\right)+,\left(\mathrm{CH}_{4}\right)+$, and both positive groups, respectively. The positivity to fasting GBT $\left(\mathrm{H}_{2}\right)$ among the GBT + patients of cholecystectomy group was $76 \%$ (22 of 29), as diagnosed by the elevated fasting $\mathrm{H}_{2}$ level, while only $24 \%$ (7 of 29) showed increase in the breath $\mathrm{H}_{2}$ concentration of more than 12 ppm above the baseline value within 60 minutes. Among cholecystectomy patients, the scores of frequency, bothersomeness, and total symptoms were significantly higher in GBT+ patients than in GBT - patients (Table 2). The GBT + group had high-

Table 2. Characteristics of the Cholecystectomy Patients According to the Positivity to Glucose Breath Test

\begin{tabular}{|c|c|c|c|c|}
\hline & \multicolumn{2}{|c|}{ Glucose breath test } & \multirow{2}{*}{$\begin{array}{l}L \text {-test }{ }^{\mathrm{a}} \\
P \text {-value }\end{array}$} & \multirow{2}{*}{$P$-value } \\
\hline & Positive $(\mathrm{n}=29)$ & Negative $(\mathrm{n}=33)$ & & \\
\hline Age (year) & $54.38 \pm 16.75$ & $52.30 \pm 14.41$ & 0.722 & 0.602 \\
\hline Gender (male) & $18(55 \%)$ & $19(66 \%)$ & & 0.378 \\
\hline $\mathrm{BMI}\left(\mathrm{kg} / \mathrm{m}^{2}\right)$ & $24.55 \pm 3.68$ & $24.17 \pm 3.76$ & 0.645 & 0.686 \\
\hline Diagnosis of IBS & $9(31 \%)$ & $10(30 \%)$ & & 0.953 \\
\hline Total symptom score & $56.75 \pm 27.52$ & $37.85 \pm 23.80$ & 0.243 & $<0.01$ \\
\hline Frequency score & $30.38 \pm 13.09$ & $21.27 \pm 12.15$ & 0.571 & $<0.01$ \\
\hline Bothersome score & $26.38 \pm 15.09$ & $16.58 \pm 12.18$ & 0.128 & $<0.01$ \\
\hline
\end{tabular}

${ }^{a}$ Levene's test for Equality of Variances for continuous variables.

BMI, body mass index; FGID, functional gastrointestinal disorder; IBS, irritable bowel syndrome. 


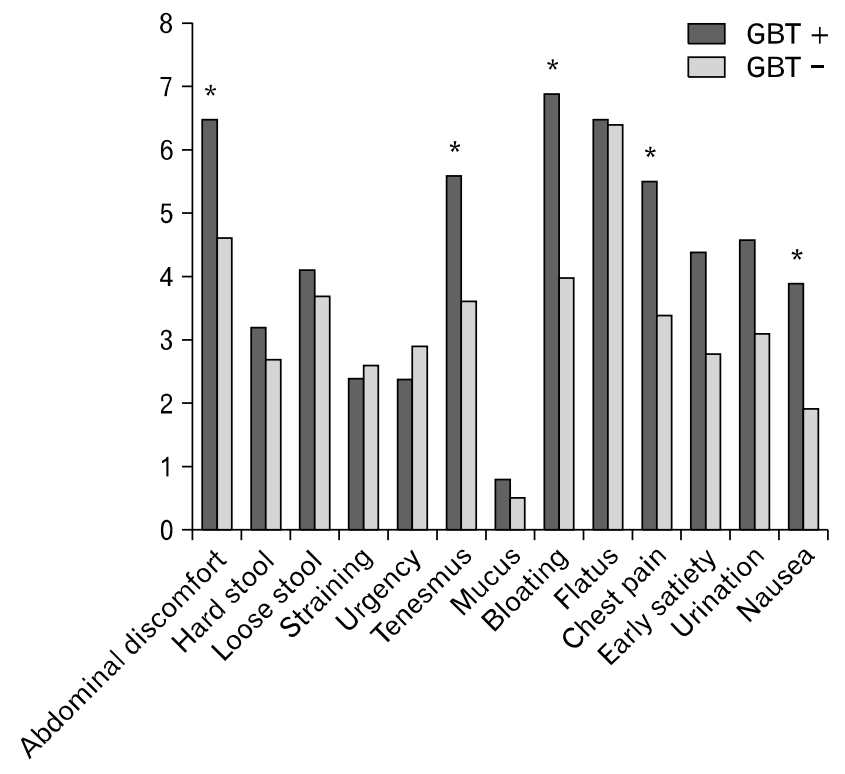

Figure 2. Total symptom scores of individual intestinal symptoms according to the positive of glucose breath test in patients with cholecystectomy. The GBT+ group had higher symptom scores of significance in abdominal discomfort, tenesmus, bloating, chest discomfort, and nausea than those of the GBT - group $\left({ }^{\star} P<0.05\right)$.

er symptom scores of significance or tendency in abdominal discomfort (6.48 \pm 3.48 vs $4.61 \pm 3.82 ; P=0.047)$, tenesmus (5.59 3.67 vs $3.6 \pm 12.66 ; P=0.017)$, bloating $(6.93 \pm 3.75$ vs $4.03 \pm 4.10 ; P<0.01)$, chest discomfort (5.52 \pm 4.39 vs 3.42 $\pm 3.16 ; P=0.038)$, early satiety (4.41 \pm 3.76 vs $2.82 \pm 2.88 ; P$ $=0.064)$ and nausea $(3.89 \pm 4.78$ vs $1.91 \pm 2.43 ; P=0.050)$ than those of the GBT - group (Fig. 2). Logistic regression analysis showed the status of cholecystectomy to be the only significant independent factor for predicting SIBO in patients with gastrointestinal symptoms (odds ratio, 2.35 ; $95 \%$ confidence interval, 1.24-4.48; $P<0.01$ ). There was no significant difference among the diagnosis of IBS, age, gender, BMI, and total symptom score.

\section{Discussion}

This study demonstrated that the positivity to GBT indicating SIBO in the post-cholecystectomy patients was high up to $46.8 \%$ compared with that in the patients with functional gastsrointestinal disorder $(26.2 \%)$ or that in historical healthy controls $(13.3 \%)$. Our findings suggest that SIBO might be one of etiology of the post cholecystectomy syndrome. Although the relationship between intestinal bacterial overgrowth and post-chol- ecystectomy state has been suggested in few epidemiological study and experimental study, ${ }^{15,16}$ the precise pathophysiology of SIBO in the post cholecystectomy syndrome still remains unclear. Conjugated bile acids are known as bacteriostatic agent, and it might contribute to the sterility of small intestinal content. Although it is still controversial several studies showed that bile acid pool size may be reduced at first after cholecystectomy, which could result in bile acid malabsorption. ${ }^{3}$ The intestinal bacterial overgrowth induces the bacterial deconjugation of bile and it should result in a further reduction in bile acid concentration because unconjugated bile acid is rapidly absorbed by nonionic diffusion. After cholecystectomy, decreased bile acid pool and bile acid malabsorption might promote bacterial growth, leading to more deconjugation and creating a vicious cycle. ${ }^{17}$

Previous studies reported that SIBO is one of the most important causes of FGID including IBS. ${ }^{18}$ However, FGID is comprised of complex symptoms and a heterogeneous disorder with a wide spectrum of symptoms including abdominal pain, bloating, flatulence, diarrhea and /or constipation etc. A systematic review and meta-analysis of studies investigating the frequency of SIBO in IBS found that the prevalence of SIBO was between $4 \%$ and $64 \% .{ }^{18}$ It depends on heterogeneity between studies, small study effects and publication bias leading to likely overestimation of the prevalence of a positive test for SIBO as well. It means that SIBO is one of the most important causes in IBS, but they are not completely the same disease. The positivity to GBT was common in patients with intestinal symptoms, even if not related to IBS. ${ }^{7}$ A significant correlation between the positivity to GBT between post-cholecystectomy group and FGID group was shown in this study, however any differences in scores of intestinal symptoms between 2 groups were not found. The causes of FGID are not only SIBO but also multiple factors such as altered gut motility, visceral hypersensitivity, abnormal brain-gut interaction, autonomic dysfunction, and immune activation. ${ }^{8}$ These various factors may affect the symptoms of FGIDs. Among post-cholecystectomy patients, the total symptom score in the GBT+ patients was $56.75 \pm 27.52$, whereas only $37.85 \pm$ 23.80 in the GBT - patients $(P<0.01)$. Our results revealed that SIBO might be an important risk factor for gastrointestinal symptoms in post-cholecystectomy patients. When all other organic diseases were excluded and breath test was performed, post-cholecystectomy patients with SIBO benefited from antibiotics therapy.

The positivity to fasting breath $\mathrm{H}_{2}$ among the GBT + postcholecystectomy group was $76 \%$ (22 of 29), as diagnosed by the 
elevated fasting $\mathrm{H}_{2}$ level. The graph of GBT in the post-cholecystectomy patients showed significant difference compared with healthy control, but no prominent rise compared with FGIDs patients at any of the single time point except baseline hydrogen level. Thereafter, the $\mathrm{H}_{2}$ concentrations increased quickly up to a higher level, and then were maintained relatively consistently. It is well known that cholecystectomy shortens gut transit by accelerating passage of the fecal bolus through the colon and it may present a frequent post-cholecystectomy diarrhea. Some investigators ascribe it to an increase in colonic bile acid contact, in keeping with the expected increase in the number of enterohepatic bile acid cycles after gallbladder removal. ${ }^{19,20} \mathrm{We}$ might suppose that shortened gut transit gave ingested substrate the less opportunity to contact intestinal bacteria, resulting in quickly and slightly increased $\mathrm{H}_{2}$ level to a higher level. The reason why our study found the relatively increased baseline $\mathrm{H}_{2}$ level in postcholecystectomy patients is unclear. The previous study in our institute suggests that the fasting $\mathrm{H}_{2}$ level may be related with the slow intestinal transit and be associated with severity of intestinal symptoms. ${ }^{21}$ Interestingly although the substrate is lactulose in previous study, the feature in the graph of breath test in the elevated fasting $\mathrm{H}_{2}$ group is similar.

The previous available studies reported that the gastroduodenal reflux and gastroesophgeal refluxs might be disrupted after cholecystectomy. It might increase the incidence of gastritis, alkaline duodenogastric reflux and gastroesophageal reflux. ${ }^{22,23}$ Based on symptom surveys, post-cholecystectomy patients had higher rate of upper GI symptoms such as abdominal pain, abdominal fullness/bloating, heartburn/chest pain, early satiety and nausea except for tenesmus. It was noteworthy that upper GI symptoms were predominant than lower GI symptoms in postcholecystectomy patients. A literature also showed that post-cholecystectomy patients had a predominance of epigastric pain and thoracic pain, and also a trend for nausea, and bloating. ${ }^{24}$ We hypothesize that the substrate of stomach, which flows backward from the small intestine due to duodenogastric reflux, reflects dominant upper GI symptoms in post-cholecystectomy patients.

There are potential limitations of this study. We used the historical controls much less than the cases. However, the positive rate of SIBO observed in control of this study was similar with normal personal data and it has been validated through our other studies. ${ }^{10}$ Another limitation was the retrospective analysis. Although we had surveyed the drug history affecting the status of SIBO in our hospital record, we cannot rule out the possibility of drug history. There is a possibility that the patient had taken the drugs at other medical institutes. Some specific foods or diet can vary the result of expired $\mathrm{H}_{2}$ and $\mathrm{CH}_{4}$, which could not be surveyed before the test.

However, all data were collected consecutively and prospectively in the same standard approach, and participants were asked for their demographic data, history of drug use by a validated questionnaire before the test to avoid the recall bias. For clinical evaluation, strict cautions were required to perform the breath test regardless of academic study as our previous studies. ${ }^{21,25}$ There are 2 representative substrates for breath test, glucose and lactulose. We used glucose as substrate for the breath test in this study. GBT can detect only proximal bacteria, as glucose is completely absorbed in proximal small bowel. Although lactulose is non-absorbable agents, there are some advantages in the use of GBT in this study. The status of cholecystectomy is related with rapid intestinal transit. During the lactulose breath test, it is difficult to distinguish SIBO from colonic bacteria in case of rapid intestinal transit, ${ }^{10,26,27}$ and importantly lactulose itself induces rapid small bowel transit. ${ }^{28}$ In addition, the advantages of breath test using glucose over lactulose as the substrate for the breath test are low false positivity and superiority of diagnosing SIBO. ${ }^{29,30}$ Therefore, our institute prefers the GBT for the patients with cholecystectomy.

To the best of our knowledge, this is the first study to investigate the relationship between post-cholecystectomy syndrome and small intestinal bacterial overgrowth by means of GBT in English. In conclusion, our study showed that the SIBO with high levels of baseline $\mathrm{H}_{2}$ might be the important etiologic factor of upper GI symptoms for post-cholecystectomy patients. It could be a new therapeutic target to manage SIBO in the upper gastrointestinal symptoms in post-cholecystectomy patients. It is clear that future studies are needed to fully understand the role of SIBO in cholecystectomy patients by demonstrating the response to antibiotic treatment of SIBO.

\section{References}

1. Jaunoo SS, Mohandas S, Almond LM. Postcholecystectomy syndrome (PCS). Int J Surg 2010;8:15-17.

2. Mechetina TA, Il'chenko AA, Lychkova AE. [Rifaximin application in the overgrowth bacterial syndrome in the small.] Eksp Klin Gastroenterol 2011;(3):93-100. [Russian]

3. Bajor A, Gillberg PG, Abrahamsson H. Bile acids: short and long term effects in the intestine. Scand J Gastroenterol 2010;45:645-664.

4. Ghoshal UC, Srivastava D, Ghoshal U, Misra A. Breath tests in the diagnosis of small intestinal bacterial overgrowth in patients with irritable bowel syndrome in comparision with quantitative upper gut as- 
pirate culture. Eur J Gastroenterol Hepatol 2014;26:753-760.

5. Corrazza GR, Menozzi MG, Strocchi A, et al. The diagnosis of small bowel bacterial overgrowth. Reliability of jejunal culture and inadequacy of breath hydrogen testing. Gastoenterology 1990;98: 302-309.

6. Ford AC, Spiegel BM, Talley NJ, Moayyedi P. Small intestinal bacterial overgrowth in irritable bowel syndrome: systematic review and meta analysis. Clin Gastroenterol Hepatol 2009;7:1279-1286.

7. Sachadeva S, Rawat AK, Reddy RS, Puri AS. Small intestinal bacterial overgrowth (SIBO) in irritable bowel syndrome: frequency and predictors. J Gastroenterol Hepatol 2011;26(suppl 3):135-138.

8. Longstreth GF, Thompson WG, Chey WD, Houghton LA, Mearin F, Spiller RC. Functional bowel disorders. Gastroenterology 2006;130:1480-1491.

9. Drossman DA. Rome III: the new criteria. Chin J Dig Dis 2006;7: 181-185.

10. Paik CN, Choi MG, Lim CH, et al. The role of small intestinal bacterial overgrowth in postgastrectomy patients. Neurogastroenterol Motil 2011;23:e191-e196.

11. Park JM, Choi MG, Oh JH, et al. Cross-cultural validation of irritable bowel syndrome quality of life in Korea. Dig Dis Sci 2006;51: 1478-1484.

12. Lee KM, Paik CN, Chung WC, Jung SH, Chang UI, Yang JM. Clinical significance of colonic diverticulosis associated with bowel symptoms. J Korean Med Sci 2010;25:1323-1329.

13. Kerlin $\mathrm{P}$, Wong L. Breath hydrogen testing in bacterial overgrowth of the small intestine. Gastroenterology 1988;95:982-988.

14. Ghoshal UC. How to interpret hydrogen breath tests. J Neurogastroenterol Motil 2011;17:312-317.

15. Kullak-Ublick GA, Paumgartner G, Berr F. Long-term effects of cholecystectomy on bile acid metabolism. Hepatology 1995;21:41-45.

16. Zuccato E, Venturi M, Di Leo G, et al. Role of bile acids and metabolic activity of colonic bacteria in increased risk. Dig Dis Sci 1993; 38:514-519.

17. Lorenzo-Zúñiga V, Bartolí R, Planas R, et al. Oral bile acids reduce bacterial overgrowth, bacterial translocation, and endotoxemia in cirrhotic rats. Hepatology 2003;37:551-557.

18. Grace E, Shaw C, Whelan K, Andreyev HJ. Review article: small in- testinal bacterial overgrowth--prevalence, clinical features, current and developing diagnostic tests, and treatment. Aliment Pharmacol Ther 2013;38:674-688.

19. Fort JM, Azpiroz F, Casellas F, Andreu J, Malagelada JR. Bowel habit after cholecystectomy: physiological changes and clinical implications. Gastroenterology 1996;111:617-622.

20. Hutcheon DF, Bayless TM, Gadacz TR. Postcholecystectomy diarrhea. JAMA 1979;241:823-824.

21. Kim EJ, Paik CN, Chung WC, Lee KM, Yang JM, Choi MG. The characteristics of the positivity to the lactulose breath test in patients. Eur J Gastroenterol Hepatol 2011;23:1144-1149.

22. Stein HJ, Feussner H, Kauer W, DeMeester TR, Siewert JR. Alkaline gastroesophageal reflux: assessment by ambulatory esophageal aspiration. Am J Surg 1994;167:163-168.

23. Hyvärinen H. Relationship of previous cholecystectomy to oesophagitis and gastroduodenal ulcers. Hepatogastroenterology 1987;34: 74-80.

24. Fein M, Bueter M, Sailer M, Fuchs KH. Effect of cholecystectomy on gastric and esophageal bile reflux in patients with upper gastrointestinal symptoms. Dig Dis Sci 2008;53:1186-1191.

25. Lee KM, Paik CN, Chung WC, Yang JM, Choi MG. Breath methane positivity is more common and higher in patients with objectively proven delayed transit constipation. Eur J Gastroenterol Hepatol 2013;25:726-732.

26. Walters B, Vanner SJ. Detection of bacterial overgrowth in IBS using the lactulose $\mathrm{H}$ breath test: comparison with $\mathrm{C}-\mathrm{D}$-xylose and healthy controls. Am J Gastroenterol 2005;100:1566-1570.

27. Riordan SM, McIver CJ, Walker BM, Duncombe VM, Bolin TD, Thomas MC. The lactulose breath hydrogen test and small intestinal bacterial overgrowth. Am J Gastroenterol 1996;91:1795-1803.

28. Bond JH Jr, Levitt MD, Prentiss R. Investigation of small bowel transit time in man utilizing pulmonary hydrogen $\left(\mathrm{H}_{2}\right)$ measurements. J Lab Clin Med 1975;85:546-555.

29. SimrénM, Stotzer PO. Use and abuse of hydrogen breath tests. Gut 2006;55:297-303.

30. Sellin JH, Hart R. Glucose malabsorption associated with rapid intestinal transit. Am J Gastroenterol 1992;87:584-589. 